\title{
Assemblage of fish species associated with aquatic macrophytes in Porto Murtinho Pantanal, Mato Grosso do Sul, Brazil
}

\author{
Yzel Rondon Súarez $z^{1,3}$, Fabiane Silva Ferreira ${ }^{1}$ \& Karina Keyla Tondato ${ }^{2}$ \\ ${ }^{1}$ Laboratório de Ecologia, Centro Integrado de Análise e Monitoramento Ambiental-CInAM, \\ Universidade Estadual de Mato Grosso do Sul-UEMS, Rod. Dourados-Itahum Km 1, \\ CEP 79804-970, Dourados, MS, Brasil \\ ${ }^{2}$ Programa de Pós-graduação em Biologia Animal, Departamento de Zoologia, Universidade Federal do \\ Rio Grande Sul-UFRGS, Av. Bento Gonçalves, 9500, CEP 91501-970, Porto Alegre, RS, Brasil \\ ${ }^{3}$ Corresponding author: Yzel Rondon Súarez, e-mail: yzel@uems.br
}

SUÁREZ, Y.R., FERREIRA, F.S. \& TONDATO, K.K. Assemblage of fish species associated with aquatic macrophytes in Porto Murtinho Pantanal, Mato Grosso do Sul, Brazil. Biota Neotrop. 13(2): http://www. biotaneotropica.org.br/v13n2/en/abstract?inventory+bn02313022013

Abstract: The Porto Murtinho Pantanal lies at the limit of the Upper Paraguay River basin, and despite its biogeographical importance, several aspects of the ecology of the fish assemblage are not known. Monthly samples of fish associated with aquatic macrophytes were taken from February 2009 through January 2011. A total of 46,327 individuals were collected, belonging to 144 species. Characiformes and Siluriformes were the predominant orders, and Odontostilbe pequira, O. paraguayensis and Bryconamericus exodon were the most abundant species. Two species, Cynopotamus sp. and Pimelodus mysteriosus, were recorded for the first time in the Pantanal, and a new occurrence record of Cichla piquiti was observed, representing an approximately $400 \mathrm{~km}$ range expansion in your distribution area in the Pantanal.

Keywords: small fish, Paraguay river, fish inventories.

SUÁREZ, Y.R., FERREIRA, F.S. \& TONDATO, K.K. Assembleia de espécies de peixes associadas com macrófitas aquáticas no Pantanal de Porto Murtinho, Mato Grosso do Sul, Brasil. Biota Neotrop. 13(2): http://www.biotaneotropica.org.br/v13n2/pt/abstract?inventory+bn02313022013

Resumo: O Pantanal de Porto Murtinho está no limite da Bacia do Alto Paraguai, e apesar de sua importância biogeográfica, alguns aspectos da ecologia das assembleias de peixes não são conhecidas. Amostras mensais de peixes associados a macrófitas aquáticas foram realizadas de Fevereiro de 2009 até Janeiro de 2011. Um total de 46.327 indivíduos foram coletados, pertencentes a 144 espécies. Characiformes e Siluriformes foram as ordens predominantes, e Odontostilbe pequira, O. paraguayensis e Bryconamericus exodon foram as espécies mais abundantes. Duas espécies, Cynopotamus sp. e Pimelodus mysteriosus, foram registrados pela primeira vez no Pantanal, e uma nova ocorrência de Cichla piquiti foi observada, o que representa uma expansão de cerca de $400 \mathrm{~km}$ de alcance em sua area de distribuição no Pantanal.

Palavras-chave: peixes de pequeno porte, rio Paraguai, inventário de peixes. 


\section{Introduction}

Floodplain environments support higher taxonomic and functional diversity (Welcomme 1985, Lowe-McConnell 1999), in response to the widely varying spatial and temporal dynamics of aquatic, terrestrial and ecotone habitats (Ward et al. 1999, Robinson et al. 2002). Macrophyte beds are fundamentally important for the initial development of many fish species (Delariva et al. 1994, SánchezBotero \& Araújo-Lima 2001), in addition to acting as feeding sites (Casatti et al. 2003) and a dispersal mechanism for small-sized species (Oliver \& McKaye 1982, Machado-Allison 1990, Bulla et al. 2011). The abundance and complexity of macrophyte beds are among the main determinants of fish communities in both lentic (Súarez et al. 2001, 2004, Petry et al. 2003, Pelicice et al. 2005, Gomes et al. 2012) and lotic habitats (Bulla et al. 2011).

In the Pantanal, few and spatially poorly distributed analyses of fish communities have been published. Some studies have been carried out in the northern Pantanal (Baginski et al. 2007, Pacheco \& Silva 2009, Fernandes et al. 2010, Milani et al. 2010, Silva et al. 2010, Lourenço et al. 2012) and the central region (Súarez et al. 2001, 2004); however few data are available from the southern Pantanal. This study aimed to characterize the composition of fish species associated with aquatic macrophytes in Porto Murtinho Pantanal, near the limit of the Upper Paraguay River.

\section{Material and Methods}

\section{Study area}

Porto Murtinho is an extreme southern portion of the Pantanal, defining the southern limit of the Upper Paraguay River Basin and comprising approximately $2.8 \%$ of the total Pantanal area (Silva \& Abdon 1998). The predominant vegetation is savanna steppe (Chaco). The river reaches its highest level in June and July, and its lowest level from November through January.

\section{Sampling}

Samples were collected monthly from February 2009 through January 2011, in beds of aquatic macrophytes along the banks of the Paraguay and Amonguijá rivers and in the floodplain lakes Criminosa and Flores (Figure 1). Fish were sampled using a sieve $(0.8 \times 1.2 \mathrm{~m})$, drag nets $(1.5 \times 5.0 \mathrm{~m})$ with $2 \mathrm{~mm}$ mesh, and seines $(1.5 \times 10 \mathrm{~m})$ with 15,20 and $30 \mathrm{~mm}$ between adjacent knots. A total of 109 sample units were obtained, with non-standardized sampling effort. The scientific samples were authorized by the Instituto Brasileiro do Meio Ambiente e dos Recursos Naturais Renováveis (IBAMA/SISBIO \# 13458-1).

Fishes were fixed in $10 \%$ formalin and preserved in $70 \%$ ethanol for identification and counts. In the laboratory, identification was carried out with the help of the identification key to the Fishes of the Pantanal (Britski et al. 2007). Voucher specimens were catalogued in

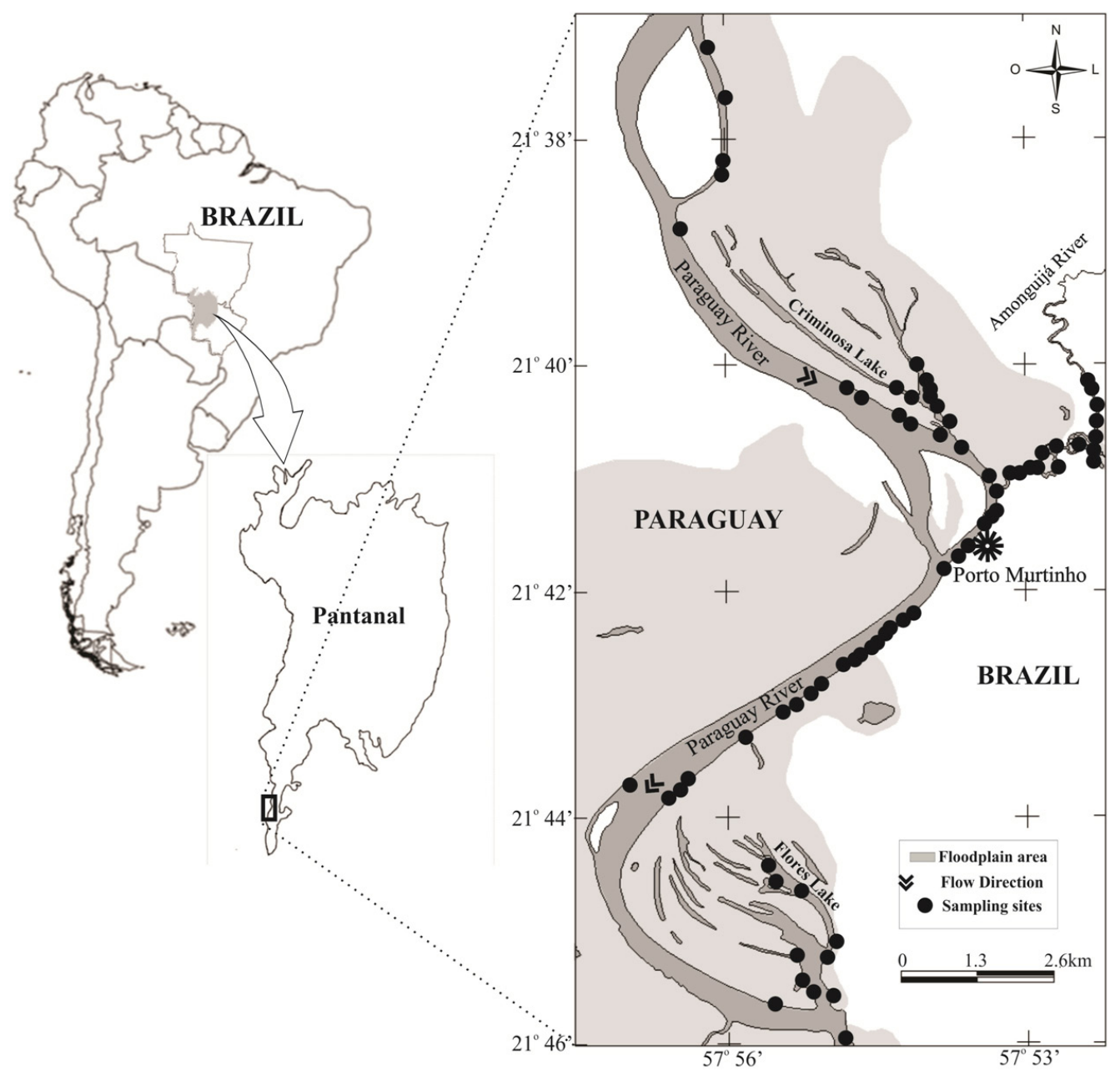

Figure 1. Study area with sampling localities at Porto Murtinho Pantanal, Mato Grosso do Sul, Brazil, from February 2009 through January 2011. 
the collection of fishes of the Department of Zoology, Universidade Federal do Rio Grande do Sul, Porto Alegre, and in the Coleção de Peixes do Nupelia, Universidade Estadual de Maringá, Maringá, Brazil.

The richness per order in each locality was compared using a chi-square test to verify any differences in the general pattern of taxonomic composition among localities.

\section{Results}

A total of 46,327 specimens were collected, belonging to 144 species in nine orders. The Characiformes showed the highest richness, with 73 species $(50.7 \%)$, followed by Siluriformes with $39(27.1 \%)$, Perciformes with 16 (11.1\%), Gymnotiformes with $10(6.9 \%)$ and Cyprinodontiformes with 2 (1.4\%). Other orders (Lepidosireniformes, Rajiformes, Synbranchiformes and Beloniformes) had one species each (Table 1). The chi-square test showed that no significant variation (chi-square $=5.85 ; \mathrm{df}=12 ; \mathrm{p}=0.92$ ) in richness per order existed among the localities (Figure 2).

The most abundant species were $O$. pequira $(10,259$ individuals/22.14\%), followed by $O$. paraguayensis $(8,617$ individuals/18.60\%) and $B$. exodon (4,275 individuals/9.23\%). In

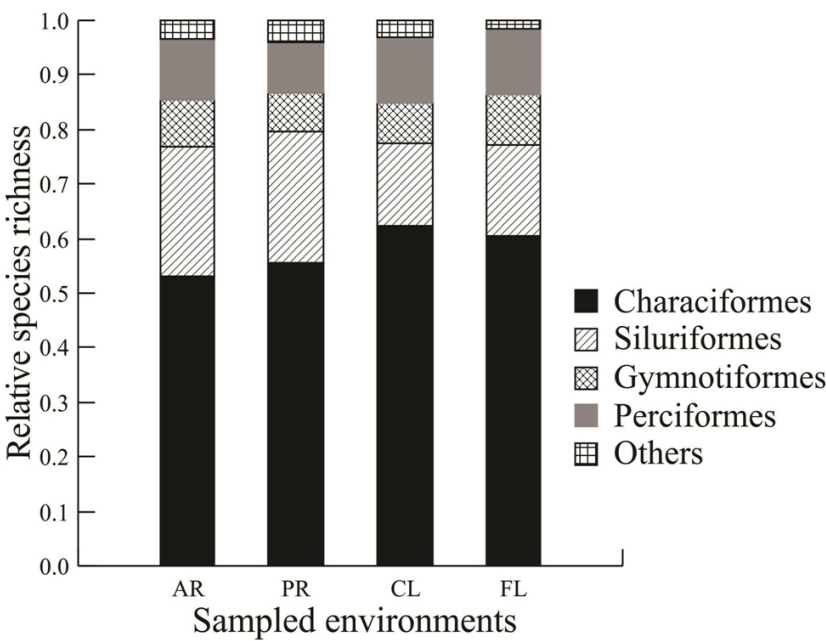

Figure 2. Relative richness per order in localities sampled at Porto Murtinho Pantanal, Mato Grosso do Sul, Brazil, from February 2009 through January 2011. $\mathrm{AR}=$ Amonguijá River; $\mathrm{PR}=$ Paraguay River; $\mathrm{CL}=$ Criminosa Lake; $\mathrm{FL}=$ Flores Lake.

Table 1. List of species and occurrences in localities sampled in Porto Murtinho Pantanal from February 2009 through January 2011. AR=Amonguijá River; $\mathrm{PR}=$ Paraguay River; $\mathrm{CL}=$ Criminosa Lake; $\mathrm{FL}=$ Flores Lake.

\begin{tabular}{|c|c|c|c|c|}
\hline Species & $\mathbf{A R}$ & PR & $\mathbf{C L}$ & FL \\
\hline \multicolumn{5}{|l|}{ Rajiformes } \\
\hline \multicolumn{5}{|l|}{ Potamotrygonidae } \\
\hline Potamotrygon brachyura (Günther, 1880) & - & $\mathrm{X}$ & - & - \\
\hline \multicolumn{5}{|l|}{ Lepidosireniformes } \\
\hline \multicolumn{5}{|l|}{ Lepidosirenidae } \\
\hline Lepidosiren paradoxa Fitzinger, 1837 & - & $\mathrm{X}$ & - & - \\
\hline \multicolumn{5}{|l|}{ Characiformes } \\
\hline \multicolumn{5}{|l|}{ Anastomidae } \\
\hline Abramites hypselonotus (Günther, 1868) & $\mathrm{X}$ & $\mathrm{X}$ & $\mathrm{X}$ & $\mathrm{X}$ \\
\hline Leporinus friderici (Bloch, 1794) & $\mathrm{X}$ & $\mathrm{X}$ & $\mathrm{X}$ & $\mathrm{X}$ \\
\hline Leporinus lacustris Campos, 1945 & $\mathrm{X}$ & $\mathrm{X}$ & $\mathrm{X}$ & $\mathrm{X}$ \\
\hline Leporinus macrocephalus Garavello and Britski, 1988 & $\mathrm{X}$ & $\mathrm{X}$ & $\mathrm{X}$ & $\mathrm{X}$ \\
\hline Leporinus obtusidens (Valenciennes, 1836) & - & $\mathrm{X}$ & - & - \\
\hline Leporinus striatus Kner, 1858 & $\mathrm{X}$ & $\mathrm{X}$ & $\mathrm{X}$ & $\mathrm{X}$ \\
\hline Leporinus sp. & $\mathrm{X}$ & $\mathrm{X}$ & $\mathrm{X}$ & $\mathrm{X}$ \\
\hline Schizodon borellii (Boulenger, 1900) & $\mathrm{X}$ & $\mathrm{X}$ & $\mathrm{X}$ & $\mathrm{X}$ \\
\hline Schizodon isognathus Kner, 1858 & - & $\mathrm{X}$ & - & - \\
\hline \multicolumn{5}{|l|}{ Characidae } \\
\hline Acestrorhynchus pantaneiro Menezes, 1992 & $\mathrm{X}$ & $\mathrm{X}$ & $\mathrm{X}$ & - \\
\hline Aphyocharax dentatus Eigenmann and Kennedy, 1903 & $\mathrm{X}$ & $\mathrm{X}$ & $\mathrm{X}$ & $\mathrm{X}$ \\
\hline Aphyocharax rathbuni Eigenmann, 1907 & $\mathrm{X}$ & $\mathrm{X}$ & $\mathrm{X}$ & - \\
\hline Aphyocharax paraguayensis Eigenmann, 1915 & $\mathrm{X}$ & $\mathrm{X}$ & $\mathrm{X}$ & $\mathrm{X}$ \\
\hline Aphyocharax anisitsi Eigenmann and Kennedy, 1903 & $\mathrm{X}$ & $\mathrm{X}$ & $\mathrm{X}$ & $\mathrm{X}$ \\
\hline Astyanax asuncionensis Géry, 1972 & $\mathrm{X}$ & $\mathrm{X}$ & $\mathrm{X}$ & $\mathrm{X}$ \\
\hline Astyanax pellegrini Eigenmann, 1907 & $\mathrm{X}$ & $\mathrm{X}$ & $\mathrm{X}$ & $\mathrm{X}$ \\
\hline Astyanax marione Eigenmann, 1911 & - & $\mathrm{X}$ & - & - \\
\hline Bryconamericus exodon (Eigenmann, 1907) & $\mathrm{X}$ & $\mathrm{X}$ & $\mathrm{X}$ & $\mathrm{X}$ \\
\hline Charax leticiae Lucena, 1987 & $\mathrm{X}$ & $\mathrm{X}$ & - & - \\
\hline Clupeacharax anchoveoides Pearson, 1924 & $\mathrm{X}$ & $\mathrm{X}$ & $\mathrm{X}$ & - \\
\hline Cynopotamus sp. & - & $\mathrm{X}$ & $\mathrm{X}$ & - \\
\hline Engraulisoma taeniatum (Castro, 1981) & - & $\mathrm{X}$ & - & $\mathrm{X}$ \\
\hline Galeocharax humeralis Valenciennes, 1834 & $\mathrm{X}$ & $\mathrm{X}$ & $\mathrm{X}$ & $\mathrm{X}$ \\
\hline Gymnocorymbus ternetzi (Boulenger, 1895) & $\mathrm{X}$ & - & - & $\mathrm{X}$ \\
\hline
\end{tabular}


Table 1. Continued...

\begin{tabular}{|c|c|c|c|c|}
\hline Species & $\mathbf{A R}$ & $\mathbf{P R}$ & $\mathbf{C L}$ & FL \\
\hline Hemigrammus marginatus Ellis, 1911 & $\mathrm{X}$ & $\mathrm{X}$ & $\mathrm{X}$ & - \\
\hline Hemigrammus lunatus Durbin, 1918 & $\mathrm{X}$ & $X$ & - & - \\
\hline Hyphessobrycon eques (Steindachner, 1882) & $\mathrm{X}$ & $\mathrm{X}$ & $\mathrm{X}$ & $\mathrm{X}$ \\
\hline Jupiaba acanthogaster (Eigenmann, 1911) & - & $\mathrm{X}$ & - & - \\
\hline Markiana nigripinnis (Perugia, 1891) & $\mathrm{X}$ & $\mathrm{X}$ & $\mathrm{X}$ & - \\
\hline Metynnis maculatus (kner, 1858) & - & $\mathrm{X}$ & - & - \\
\hline Mylossoma paraguayensis Norman, 1928 & $\mathrm{X}$ & $\mathrm{X}$ & $\mathrm{X}$ & - \\
\hline Moenkhausia forestii Benine, Mariguela and Oliveira, 2009 & $\mathrm{X}$ & $\mathrm{X}$ & $\mathrm{X}$ & $\mathrm{X}$ \\
\hline Moenkhausia sanctafilomenae (Steindachner, 1907) & $X$ & $X$ & $X$ & $\mathrm{X}$ \\
\hline Moenkhausia dichroura (Kner, 1858) & $\mathrm{X}$ & $\mathrm{X}$ & $\mathrm{X}$ & $\mathrm{X}$ \\
\hline Moenkhausia intermedia Eigenmann, 1908 & $\mathrm{X}$ & $\mathrm{X}$ & - & - \\
\hline Myloplus levis (Eigenmann and McAtee, 1907) & $\mathrm{X}$ & $\mathrm{X}$ & $\mathrm{X}$ & - \\
\hline Odontostilbe pequira (Steindachner, 1882) & $\mathrm{X}$ & $\mathrm{X}$ & $\mathrm{X}$ & $\mathrm{X}$ \\
\hline Odontostilbe paraguayensis Eigenmann and Kennedy, 1903 & $\mathrm{X}$ & $\mathrm{X}$ & $\mathrm{X}$ & $\mathrm{X}$ \\
\hline Piabucus melanostoma Holmberg, 1891 & $\mathrm{X}$ & $\mathrm{X}$ & $\mathrm{X}$ & - \\
\hline Poptella paraguayensis (Eigenmann, 1907) & $\mathrm{X}$ & $\mathrm{X}$ & $\mathrm{X}$ & - \\
\hline Prinobrama paraguayensis (Eigenmann, 1914) & $\mathrm{X}$ & $\mathrm{X}$ & $\mathrm{X}$ & $\mathrm{X}$ \\
\hline Psellogrammus kennedyi (Eigenmann, 1903) & $\mathrm{X}$ & $\mathrm{X}$ & $\mathrm{X}$ & $\mathrm{X}$ \\
\hline Pygocentrus nattereri Kner, 1858 & - & $\mathrm{X}$ & - & - \\
\hline Roeboides microlepis Steindachner, 1879 & $\mathrm{X}$ & $\mathrm{X}$ & - & $\mathrm{X}$ \\
\hline Roeboides prognatus Boulenger, 1895 & $X$ & $\mathrm{X}$ & $X$ & - \\
\hline Roeboides descalvadensis Fowler, 1932 & $\mathrm{X}$ & $X$ & $\mathrm{X}$ & $\mathrm{X}$ \\
\hline Salminus brasiliensis (Cuvier, 1816) & - & $\mathrm{X}$ & - & - \\
\hline Serrapinus calliura (Boulenger, 1900) & $\mathrm{X}$ & $\mathrm{X}$ & $\mathrm{X}$ & $\mathrm{X}$ \\
\hline Serrasalmus maculatus Kner, 1858 & $\mathrm{X}$ & $\mathrm{X}$ & $\mathrm{X}$ & - \\
\hline Serrasalmus marginatus Valenciennes, 1837 & $\mathrm{X}$ & $\mathrm{X}$ & $\mathrm{X}$ & $\mathrm{X}$ \\
\hline Tetragonopterus argenteus (Cuvier, 1816) & $X$ & $\mathrm{X}$ & $X$ & $\mathrm{X}$ \\
\hline Triportheus pantanensis (Kner, 1858) & $\mathrm{X}$ & $\mathrm{X}$ & $\mathrm{X}$ & $\mathrm{X}$ \\
\hline Triportheus nematurus (Günther, 1874) & $\mathrm{X}$ & $\mathrm{X}$ & $\mathrm{X}$ & $\mathrm{X}$ \\
\hline \multicolumn{5}{|l|}{ Crenuchidae } \\
\hline Characidium aff. zebra Eigenmann, 1909 & $\mathrm{X}$ & $X$ & $\mathrm{X}$ & $\mathrm{X}$ \\
\hline Characidium laterale (Boulenger, 1895) & $\mathrm{X}$ & $\mathrm{X}$ & $\mathrm{X}$ & $\mathrm{X}$ \\
\hline \multicolumn{5}{|l|}{ Curimatidae } \\
\hline Curimatella dorsalis (Eigenmann and Eigenmann, 1889) & $\mathrm{X}$ & $\mathrm{X}$ & $\mathrm{X}$ & $\mathrm{X}$ \\
\hline Cyphocharax gillii (Eigenmann and Kennedy, 1903) & $\mathrm{X}$ & $\mathrm{X}$ & $\mathrm{X}$ & $\mathrm{X}$ \\
\hline Psectrogaster curviventris Eigenmann and Kennedy, 1903 & $\mathrm{X}$ & $\mathrm{X}$ & $\mathrm{X}$ & - \\
\hline Potamorhina squamoralevis (Braga and Azpelicueta, 1983) & $\mathrm{X}$ & $\mathrm{X}$ & $\mathrm{X}$ & - \\
\hline Steindachnerina brevipinna (Eigenmann and Eigenmann, 1889) & $X$ & $X$ & $X$ & - \\
\hline Steindachnerina conspersa (Holmberg, 1891) & $\mathrm{X}$ & $\mathrm{X}$ & $\mathrm{X}$ & - \\
\hline Steindachnerina nigrotaenia (Boulenger, 1902) & $\mathrm{X}$ & $\mathrm{X}$ & $\mathrm{X}$ & - \\
\hline \multicolumn{5}{|l|}{ Cynodontidae } \\
\hline Rhaphiodon vulpinus Spix and Agassiz, 1829 & $\mathrm{X}$ & $\mathrm{X}$ & $\mathrm{X}$ & - \\
\hline \multicolumn{5}{|l|}{ Engraulididae } \\
\hline Lycengraulis sp. & $X$ & $\mathrm{X}$ & $X$ & $\mathrm{X}$ \\
\hline \multicolumn{5}{|l|}{ Erythrinidae } \\
\hline Hoplias sp. & - & $\mathrm{X}$ & - & - \\
\hline Hoplias malabaricus (Bloch, 1794) & $\mathrm{X}$ & $\mathrm{X}$ & $\mathrm{X}$ & $\mathrm{X}$ \\
\hline Hoplerythrinus unitaeniatus (Spix, 1829) & $\mathrm{X}$ & - & - & - \\
\hline \multicolumn{5}{|l|}{ Gasteropelecidae } \\
\hline Gasteropelecus sternicla (Linnaeus, 1758) & - & $\mathrm{X}$ & $X$ & - \\
\hline Thoracocharax stellatus (Kner, 1858) & $\mathrm{X}$ & $\mathrm{X}$ & $\mathrm{X}$ & - \\
\hline \multicolumn{5}{|l|}{ Hemiodontidae } \\
\hline Hemiodus orthonops (Eigenmann and Kennedy, 1903) & $\mathrm{X}$ & $\mathrm{X}$ & $\mathrm{X}$ & $\mathrm{X}$ \\
\hline Lebiasinidae & & & & \\
\hline
\end{tabular}


Table 1. Continued...

\begin{tabular}{|c|c|c|c|c|}
\hline Species & $\mathbf{A R}$ & PR & $\mathbf{C L}$ & FL \\
\hline Pyrrhulina australis Eigenmann and Kennedy, 1903 & $\mathrm{X}$ & $\mathrm{X}$ & $\mathrm{X}$ & $\mathrm{X}$ \\
\hline \multicolumn{5}{|l|}{ Paradontidae } \\
\hline Apareiodon affinis (Steindachner, 1879) & $\mathrm{X}$ & $\mathrm{X}$ & $\mathrm{X}$ & - \\
\hline \multicolumn{5}{|l|}{ Prochilodontidae } \\
\hline Prochilodus lineatus (Valenciennes, 1836) & $\mathrm{X}$ & $\mathrm{X}$ & $\mathrm{X}$ & $\mathrm{X}$ \\
\hline \multicolumn{5}{|l|}{ Siluriformes } \\
\hline \multicolumn{5}{|l|}{ Auchenipteridae } \\
\hline Auchenipterus nigripinnis (Boulenger, 1895) & $\mathrm{X}$ & $\mathrm{X}$ & - & - \\
\hline Epapterus dispilurus Cope, 1878 & - & $\mathrm{X}$ & - & - \\
\hline Parauchenipterus striatulus (Steindachner, 1876) & $\mathrm{X}$ & - & - & $\mathrm{X}$ \\
\hline Parauchenipterus galeatus (Linnaeus, 1766) & $\mathrm{X}$ & $\mathrm{X}$ & $\mathrm{X}$ & $\mathrm{X}$ \\
\hline \multicolumn{5}{|l|}{ Aspredinidae } \\
\hline Bunocephalus doriae Boulenger, 1902 & $\mathrm{X}$ & $\mathrm{X}$ & $\mathrm{X}$ & $\mathrm{X}$ \\
\hline \multicolumn{5}{|l|}{ Callichthyidae } \\
\hline Callichthys callichthys (Linnaeus, 1758) & - & - & $\mathrm{X}$ & - \\
\hline Corydoras hastatus Eigenmann and Eigenmann, 1888 & $\mathrm{X}$ & $\mathrm{X}$ & $\mathrm{X}$ & $\mathrm{X}$ \\
\hline Hoplosternum littorale (Hancock, 1828) & $\mathrm{X}$ & - & - & - \\
\hline Leplosternum pectorale (Boulenger, 1895) & $\mathrm{X}$ & - & - & $\mathrm{X}$ \\
\hline \multicolumn{5}{|l|}{ Doradidae } \\
\hline Platydoras armatulus (Valenciennes, 1840) & $\mathrm{X}$ & - & - & - \\
\hline Oxydoras kneri Bleeker, 1862 & - & $\mathrm{X}$ & - & - \\
\hline \multicolumn{5}{|l|}{ Heptapteridae } \\
\hline Rhamdia sp. Bleeker, 1858 & $\mathrm{X}$ & $\mathrm{X}$ & $\mathrm{X}$ & - \\
\hline \multicolumn{5}{|l|}{ Loricariidae } \\
\hline Farlowella paraguayensis Retzer and Page, 1997 & $\mathrm{X}$ & $\mathrm{X}$ & - & - \\
\hline Hemiodontichthys acipenserinus (Kner, 1853) & $\mathrm{X}$ & $\mathrm{X}$ & - & - \\
\hline Hypoptopoma inexspectatum (Holmberg, 1893) & $\mathrm{X}$ & $\mathrm{X}$ & $\mathrm{X}$ & $\mathrm{X}$ \\
\hline Hypostomus sp. Lacépède, 1803 & $\mathrm{X}$ & $\mathrm{X}$ & $\mathrm{X}$ & $\mathrm{X}$ \\
\hline Hypostomus boulengeri (Eigenmann and Kennedy, 1903) & $\mathrm{X}$ & $\mathrm{X}$ & - & - \\
\hline Hypostomus latifrons Weber, 1986 & $\mathrm{X}$ & $\mathrm{X}$ & - & - \\
\hline Loricariichthys platymetopon Isbrücker and Nijssen, 1979 & $\mathrm{X}$ & $\mathrm{X}$ & $\mathrm{X}$ & - \\
\hline Loricariichthys labialis (Boulenger, 1895) & - & $\mathrm{X}$ & $\mathrm{X}$ & - \\
\hline Loricaria sp. Linnaeus, 1758 & $\mathrm{X}$ & $\mathrm{X}$ & - & - \\
\hline Otocinclus vittatus Regan, 1904 & $\mathrm{X}$ & $\mathrm{X}$ & $\mathrm{X}$ & $\mathrm{X}$ \\
\hline Pterygoplichthys ambrosettii (Holmberg, 1893) & - & $\mathrm{X}$ & - & - \\
\hline Pyxiloricaria menezesi Isbücker and Nijssen, 1984 & $\mathrm{X}$ & $\mathrm{X}$ & - & $\mathrm{X}$ \\
\hline Rineloricaria parva (Boulenger, 1895) & $\mathrm{X}$ & $\mathrm{X}$ & $\mathrm{X}$ & $\mathrm{X}$ \\
\hline Sturisoma barbatum (Kner, 1853) & $\mathrm{X}$ & $\mathrm{X}$ & - & - \\
\hline \multicolumn{5}{|l|}{ Pimelodidae } \\
\hline Pimelodella taenioptera Ribeiro, 1914 & $\mathrm{X}$ & $\mathrm{X}$ & $\mathrm{X}$ & - \\
\hline Pimelodella gracilis (Valenciennes, 1840) & - & $\mathrm{X}$ & - & - \\
\hline Pimelodella mucosa Eigenmann and Ward, 1907 & - & $\mathrm{X}$ & - & - \\
\hline Pimelodus maculatus Lacépède, 1803 & $\mathrm{X}$ & $\mathrm{X}$ & $\mathrm{X}$ & $\mathrm{X}$ \\
\hline Pimelodus mysteriosus Azpelicueta, 1998 & $\mathrm{X}$ & - & - & - \\
\hline Pimelodus argenteus Perugia, 1891 & - & $\mathrm{X}$ & - & - \\
\hline Iheringichthys labrosus (Lütken, 1874) & - & $\mathrm{X}$ & - & - \\
\hline Pinirampu pirinampu (Spix and Agassiz, 1829) & - & $\mathrm{X}$ & - & - \\
\hline Megalonema platanum (Günther, 1880) & $\mathrm{X}$ & $\mathrm{X}$ & - & - \\
\hline Sorubim lima (Bloch and Schneider, 1801) & $\mathrm{X}$ & $\mathrm{X}$ & - & - \\
\hline Pseudoplatystoma corruscans (Spix and Agassiz, 1829) & $\mathrm{X}$ & $\mathrm{X}$ & - & - \\
\hline Zungaro zungaro (Humboldt and Valenciennes, 1821) & $\mathrm{X}$ & - & - & - \\
\hline \multicolumn{5}{|l|}{ Trichomycteridae } \\
\hline Ituglanis eichorniarium (Miranda Ribeiro, 1912) & - & - & $\mathrm{X}$ & - \\
\hline
\end{tabular}


Table 1. Continued...

\begin{tabular}{|c|c|c|c|c|}
\hline Species & $\mathbf{A R}$ & PR & $\mathbf{C L}$ & FL \\
\hline \multicolumn{5}{|l|}{ Gymnotiformes } \\
\hline \multicolumn{5}{|l|}{ Apternotidae } \\
\hline Apteronotus albifrons (Linnaeus, 1766) & $\mathrm{X}$ & $\mathrm{X}$ & - & $\mathrm{X}$ \\
\hline \multicolumn{5}{|l|}{ Gymnotidae } \\
\hline Gymnotus spp. & $\mathrm{X}$ & $\mathrm{X}$ & $\mathrm{X}$ & - \\
\hline Gymnotus inaquilabiatus (Valenciennes, 1839) & $\mathrm{X}$ & $\mathrm{X}$ & $\mathrm{X}$ & - \\
\hline Gymnotus paraguensis Albert and Crampton, 2003 & $\mathrm{X}$ & $\mathrm{X}$ & - & - \\
\hline \multicolumn{5}{|l|}{ Hypopomidae } \\
\hline Brachyhypopomus sp. A Mago Leccia, 1994 & $\mathrm{X}$ & - & $\mathrm{X}$ & - \\
\hline Brachyhypopomus sp. B Mago Leccia, 1994 & $\mathrm{X}$ & $\mathrm{X}$ & $\mathrm{X}$ & $\mathrm{X}$ \\
\hline Brachyhypopomus sp. C Mago Leccia, 1994 & $\mathrm{X}$ & $\mathrm{X}$ & $\mathrm{X}$ & $\mathrm{X}$ \\
\hline \multicolumn{5}{|l|}{ Sternopygidae } \\
\hline Sternopygus macrurus (Bloch and Schneider, 1801) & $\mathrm{X}$ & $\mathrm{X}$ & $\mathrm{X}$ & $\mathrm{X}$ \\
\hline Eigenmannia trilineata López and Castelo, 1966 & $\mathrm{X}$ & $\mathrm{X}$ & $\mathrm{X}$ & $\mathrm{X}$ \\
\hline Eigenmannia virescens (Valenciennes, 1842) & $\mathrm{X}$ & $\mathrm{X}$ & - & $\mathrm{X}$ \\
\hline \multicolumn{5}{|l|}{ Perciformes } \\
\hline \multicolumn{5}{|l|}{ Cichlidae } \\
\hline Aequidens plagiozonatus Kullander, 1984 & $\mathrm{X}$ & $\mathrm{X}$ & $\mathrm{X}$ & $\mathrm{X}$ \\
\hline Apistogramma trifasciata (Eigenmann and Kennedy, 1903) & $\mathrm{X}$ & $\mathrm{X}$ & $\mathrm{X}$ & $\mathrm{X}$ \\
\hline Apistogramma commbrae (Regan, 1906) & $\mathrm{X}$ & $\mathrm{X}$ & $\mathrm{X}$ & $\mathrm{X}$ \\
\hline Apistogramma borellii (Regan, 1906) & $\mathrm{X}$ & $\mathrm{X}$ & $\mathrm{X}$ & $\mathrm{X}$ \\
\hline Astronotus crassipinnis Heckel, 1840 & $\mathrm{X}$ & - & $\mathrm{X}$ & - \\
\hline Bujurquina vittata (Heckel, 1840) & $\mathrm{X}$ & $\mathrm{X}$ & $\mathrm{X}$ & $\mathrm{X}$ \\
\hline Chaetobranchopsis australis Eigenmann and Ward, 1907 & $\mathrm{X}$ & $\mathrm{X}$ & $\mathrm{X}$ & - \\
\hline Cichla piquiti Kullander and Ferreira, 2006 & - & $\mathrm{X}$ & - & - \\
\hline Cichlasoma dimerus (Heckel, 1840) & $\mathrm{X}$ & - & - & - \\
\hline Crenicichla semifasciata (Heckel, 1840) & $\mathrm{X}$ & $\mathrm{X}$ & $\mathrm{X}$ & $\mathrm{X}$ \\
\hline Crenicichla lepidota Heckel, 1840 & $\mathrm{X}$ & $\mathrm{X}$ & $\mathrm{X}$ & $\mathrm{X}$ \\
\hline Crenicichla vittata Heckel, 1840 & $\mathrm{X}$ & $\mathrm{X}$ & $\mathrm{X}$ & - \\
\hline Gymnogeophagus balzanii (Perugia, 1891) & $\mathrm{X}$ & $\mathrm{X}$ & $\mathrm{X}$ & - \\
\hline Mesonauta festivus (Heckel, 1840) & - & - & - & $\mathrm{X}$ \\
\hline Satanoperca pappaterra $($ Heckel, 1840) & $\mathrm{X}$ & - & - & - \\
\hline \multicolumn{5}{|l|}{ Sciaenidae } \\
\hline Pachyurus bonariensis Steindachner, 1879 & - & $\mathrm{X}$ & - & - \\
\hline \multicolumn{5}{|l|}{ Beloniformes } \\
\hline \multicolumn{5}{|l|}{ Belonidae } \\
\hline Potamorhaphis eigenmanni Ribeiro, 1915 & $\mathrm{X}$ & $\mathrm{X}$ & $\mathrm{X}$ & - \\
\hline \multicolumn{5}{|l|}{ Cyprinodontiformes } \\
\hline \multicolumn{5}{|l|}{ Rivulidae } \\
\hline Rivulus punctatus Boulenger, 1895 & $\mathrm{X}$ & $\mathrm{X}$ & $\mathrm{X}$ & - \\
\hline Trigonectes balzanii (Perugia, 1891) & $\mathrm{X}$ & - & - & - \\
\hline \multicolumn{5}{|l|}{ Synbranchiformes } \\
\hline \multicolumn{5}{|l|}{ Synbranchidae } \\
\hline Synbranchus marmoratus Bloch, 1795 & $\mathrm{X}$ & $\mathrm{X}$ & $\mathrm{x}$ & $\mathrm{X}$ \\
\hline
\end{tabular}

contrast, 22 species were each represented by only one individual. Thus, the three most abundant species comprised approximately $50 \%$ of the total sampled individuals, while 123 species represented individually less than $1 \%$, and together comprised $14.14 \%$ of the total sample. Despite the predominance of small species, many of the fishes collected are important for fisheries, including P. corruscans
(Pintado), Z. zungaru (Jaú), S. brasiliensis (Dourado), and S. lima (Jurupensen), among others.

Among the species collected, the tucunare Cichla piquiti, an introduction from the Amazon basin, was found in the Paraguay River. Another, Cynopotamus sp., was recorded for the first time in the Upper Paraguay River, and its taxonomic status is unknown. 
Pimelodus mysteriosus, a species of the Paraná basin, was recorded for the first time in the Upper Paraguay River.

\section{Discussion}

The fish species associated with aquatic macrophytes in Porto Murtinho Pantanal comprised 53.5\% of the total species richness known for the Pantanal (Britski et al. 2007). This shows both the homogeneity in fish species distribution across the Pantanal floodplain, and the importance of aquatic macrophytes for maintenance of fish diversity. Moreover, the predominance of Characiformes and Siluriformes observed follows the Neotropical biogeographic pattern and other studies in the Pantanal (Willink et al. 2000, Súarez et al. 2001, Baginski et al. 2007, Pacheco \& Silva 2009, Milani et al. 2010).

The dominance of two small species of Cheirodontinae (O. pequira e O. paraguayensis) follows a habitat pattern, since other studies on fishes associated with aquatic macrophytes have yield the same general results (Súarez et al. 2001, Baginski et al. 2007, Pacheco \& Silva 2009, Silva et al. 2010). This suggests that the macrophyte habitat is favorable for these species, probably because of the good availability of food and protection from predators. Moreover, the greater dominance of a few species is common in tropical communities. Magurran \& Henderson (2003) suggested that abundant/common species are those residing in this habitat, while other species are occasional visitors. This hypothesis can be used to explain our data, in response to the close association between the species found and macrophyte beds. The smaller fish species are possibly more abundant because they are resident in the macrophyte beds.

Also, the occurrence of some commercially important species occurred as juveniles, suggesting that macrophyte beds are also an important habitat for the initial development of larger species, as demonstrated by Delariva et al. (1994) for the Upper Paraná River.

In relation to introduced specie tucunaré Cichla piquiti, Resende et al. (2008) defines as a well-established species in the Pantanal, and reported its occurrence from the Piquiri River (northern Pantanal) to the region of Corumbá (central Pantanal). Our data revealed that it occurs approximately $400 \mathrm{~km}$ southward, at the limit of the Pantanal (Upper Paraguay River). Therefore, along the main river (the Paraguay), the tucunaré occupies approximately $70 \%$ of the north-south axis of the Pantanal, although its occurrence in tributaries of the Paraguay River has not been evaluated, nor has its influence on aquatic communities.

The specimens of Cynopotamus sp. collected are of unknown taxonomic status; they differ from others reported from the Pantanal, although they may have been recorded in the Middle Paraguay River. Only after careful analysis can we can determine if this record is a geographical expansion of distribution, or an undescribed species. Complementarily, $P$. mysteriosus is a species described from the Paraná basin, and is here first recorded in the Pantanal.

In conclusion, the fish diversity associated with aquatic macrophytes in Porto Murtinho Pantanal is composed by small-sized species, with some juveniles and adults of larger species occurring in different frequencies. Some species were recorded for the first time in the Pantanal (Cynopotamus sp. and P. mysteriosus), or the record represents an expansion of the known distribution (e.g., C. piquiti). Finally, the great fish diversity, typical of the Pantanal, suggests the fundamental importance of macrophyte beds for fish and fisheries ecology.

\section{Acknowledgments}

The authors gratefully acknowledge the Centro de Pesquisa do Pantanal (CPP/MCT), Fundect, UEMS for their financial, personnel, and logistical support. We are indebted to Ediléia A. Silva, Maiane J. Pereira, Mariane I. Santos, Marcelo M. Souza, Marlon C. Pereira, Gabriela S. V. Duarte, Patricia L. Rondon and William F. Antonialli Júnior for help in the field work. Y. R. Súarez is supported by productivity grants from $\mathrm{CNPq}$.

\section{References}

BAGINSKI, L.J., FLORENTINO, A.C., FERNANDES, I.M., PENHA, J.M.F \& MATEUS, L.A.F. 2007. A dimensão espacial e temporal da diversidade de peixes da zona litoral vegetada de lagoas marginais da planície de inundação do rio Cuiabá, Pantanal, Brasil. Biota Neotrop. 7(3): http:// www.biotaneotropica.org.br/v7n3/pt/abstract?article+bn04007032007 (último acesso em 27/08/2012).

BRITSKI, H.A., SILIMON, K.Z. \& LOPES, B.S. 2007. Peixes do Pantanal: Manual de Identificação. 2. ed. Embrapa, Brasília, 227p.

BULLA, C.K., GOMES, L.C., MIRANDA, L.E. \& AGOSTINHO, A.A. 2011. The ichthyofauna of drifting macrophyte mats in the Ivinhema River, upper Paraná River basin, Brazil. Neotrop. Ichthyol. 9(2):403-409. http:// dx.doi.org/10.1590/S1679-62252011005000021

CASATTI, L., MENDES, H.F. \& FERREIRA, K.M. 2003. Aquatic macrophytes as feeding site for small fishes in the Rosana Reservoir, Paranapanema River, Southeastern Brazil. Braz. J. Biol. 63(2):213-222. http://dx.doi.org/10.1590/S1519-69842003000200006

DELARIVA, R.L.,AGOSTINHO,A.A., NAKATANI, K. \& BAUMGARTNER, G. 1994. Ichthyofauna associated to aquatic macrophytes in the upper Parana River floodplain. Rev. Unimar 3:41-60.

FERNANDES, I.M., MACHADO, F.A. \& PENHA, J.M.F. 2010. Spatial pattern of a fish assemblage in a seasonal tropical wetland: effects of habitat, herbaceous plant biomass, water depth, and distance from species sources. Neotrop. Ichthyol. 8(2):289-298. http://dx.doi.org/10.1590/ S1679-62252010000200007

GOMES, L.C., BULLA, C.K., AGOSTINHO, A.A., VASCONCELOS, L.P. \& MIRANDA, L.E. 2012. Fish assemblage dynamics in a Neotropical floodplain relative to aquatic macrophytes and the homogenizing effect of a flood pulse. Hydrobiologia 685:97-107. http://dx.doi.org/10.1007/ s10750-011-0870-6

LOURENÇO, L.S., FERNANDES, I.M., PENHA, J.M.F. \& MATEUS, L.A.F. 2012. Persistence and stability of cichlid assemblages in neotropical floodplain lagoons. Environ. Biol. Fish. 93:427-437. http:// dx.doi.org/10.1007/s10641-011-9933-9

LOWE-McCONNELL, R.H. 1999. Estudos Ecológicos de Comunidades de Peixes Tropicais. Edusp, São Paulo, 534p.

MACHADO-ALLISON, A. 1990. Ecologia de los peces de las areas inundables de los llanos de Venezuela. Interciencia 15:411-423.

MAGURRAN, A.E. \& HENDERSON, P.A. 2003. Explaining the excess of rare species in natural species abundance distributions. Nature 422:714-716. http://dx.doi.org/10.1038/nature01547

MILANI, V., MACHADO, F.A. \& SILVA, V.C.F. 2010. Assembléias de peixes associados às macrófitas aquáticas em ambientes alagáveis do Pantanal de Poconé, MT, Brasil. Biota Neotrop. 10(2): http://www. biotaneotropica.org.br/v10n2/en/abstract?article+bn02310022010 (último acesso em 28/08/2012).

OLIVER, M.K. \& McKAYE, K.R. 1982. Floating islands - a means of fish dispersal in Lake Malawi, Africa. Copeia 1982:748-754. http://dx.doi. org/10.2307/1444082

PACHECO, E.B. \& SILVA, C.J. 2009. Fish associated with aquatic macrophytes in the Chacororé-Sinhá Mariana lake system and Mutum River, Pantanal of Mato Grosso, Brazil. Braz. J. Biol. 69(1):101-108. http://dx.doi.org/10.1590/S1519-69842009000100012 
PELiCICE, F.M., AGOSTINHO, A.A. \& THOMAZ, S.M. 2005. Fish assemblages associated with Egeria in a tropical reservoir: investigating the effects of plant biomass and diel period. Acta Oecol. 27:9-16. http:// dx.doi.org/10.1016/j.actao.2004.08.004

PETRY, P., BAYLEY, P.B. \& MARKLE, D.F. 2003. Relationships between fish assemblages, macrophytes and environmental gradients in the Amazon River Floodplain. J. Fish Biol. 63:547-579. http://dx.doi. org/10.1046/j.1095-8649.2003.00169.x

RESENDE, E.K., MARQUES, D.K. \& FERREIRA, L.K.S.G. 2008. A successful case of biological invasion: the fish Cichla piquiti, an Amazonian species introduced into the Pantanal, Brazil. Braz. J. Biol. 68(4):799-805. http://dx.doi.org/10.1590/S1519-69842008000400014

ROBINSON, C.T., TOCKNER, K. \& WARD, J.V. 2002. The fauna of dynamic riverine landscapes. Freshwater Biol. 47:661-677. http://dx.doi. org/10.1046/j.1365-2427.2002.00921.x

SÁNCHEZ-BOTERO, J.I. \& ARAÚJO-LIMA, C.A.R.M. 2001. As macrófitas aquáticas como berçário para a ictiofauna da várzea do rio Amazonas. Acta Amazon. 31(3):437-447.

SILVA, H.P., PETRY, A.C. \& SILVA, C.J. 2010. Fish communities of the Pantanal wetland in Brazil: evaluating the effects of the upper Paraguay River flood pulse on baía Caiçara fish fauna. Aquat. Ecol. 44:275-288. http://dx.doi.org/10.1007/s10452-009-9289-9

SILVA, J.S.V. \& ABDON, M.M. 1998. Delimitação do Pantanal brasileiro e suas sub-regiões. Pesqui. Agropecu. Bras. 33:1703-1711.
SÚAREZ, Y.R., PETRERE-JÚNIOR, M. \& CATELLA, A.C. 2001. Factors determining the structure of fish communities in Pantanal lagoons (MS, Brazil). Fisheries Manag. Ecol. 8(2):173-186. http://dx.doi.org/10.1046/ j.1365-2400.2001.00236.x

SÚAREZ, Y.R., PETRERE-JÚNIOR, M. \& CATELLA, A.C. 2004. Factors regulating diversity and abundance of fish communities in Pantanal lagoons, Brazil. Fisheries Manag. Ecol. 11(1):45-50. http://dx.doi. org/10.1111/j.1365-2400.2004.00347.x

WARD, J.V., TOCKNER, K. \& SCHIEMER, F. 1999. Biodiversity of floodplain river ecosystems: ecotones and connectivity. Regul. River. 15:125-139. http://dx.doi.org/10.1002/(SICI)10991646(199901/06)15:1/3\%3C125::AID-RRR523\%3E3.0.CO;2-E

WELCOMME, R.L. 1985. River Fisheries. Food and Agriculture Organization Fisheries Technical Paper, v.262, p.1-330.

WILlinK, P.W., FROEHLICH, O., MACHADO-ALISSON, A.M., MENEZES, N.A., OYAKAWA, O.T., CATELLA, A.C., CHEMOFF, B., LIMA, F.C.T., TOLEDO-PIZA, M., ORTEGA, H., ZANATA, A.M. \& BARRIGA, R. 2000. Fishes of the rios Negro, Negrinho, Taboco, Aquidauana, Taquari, and Miranda, Pantanal, Brasil: Diversity, distribution, critical habitats and value. In Biological Assessment of the Aquatic Ecosystems of the Pantanal, Mato Grosso do Sul, Brasil (P.W. Willink, B. Chemoff, L.E. Alonso, J.R. Montambault \& R.A. Lourival) Conservation International, Washington, p.63-81. RAP Bulletin of Biological Assessment. n.18. 